\title{
Trophic levels of fish species of commercial importance in the Colombian Caribbean
}

\author{
Camilo B. García \& Cristian Camilo Contreras \\ Departamento de Biología, Universidad Nacional de Colombia, Av. Cra. 30 \# 45 -03, Bogotá D.C., Colombia; \\ cbgarciar@unal.edu.co, cccontrerasg@unal.edu.co
}

Received 13-IX-2010. C Corrected 28-I-2011. $\quad$ Accepted 01-III-2011.

\begin{abstract}
Ecological studies on commercial important fish species are of great value to support resource management issues. This study calculated trophic levels of those Colombian Caribbean fish species whose diet has been locally described. Usable diet data of 119 species resulted in 164 trophic level estimates. An ordinary regression model relating trophic level and fish size was formulated. The regression slope was positive and significantly different from zero $(\mathrm{p}<0.05)$ suggesting a scaling of trophic level with fish size. Both the list of trophic levels and the regression model should be of help in the formulation of trophic indicators and models of neotropical ecosystems. Rev. Biol. Trop. 59 (3): 1195-1203. Epub 2011 September 01.
\end{abstract}

Key words: trophic level, fishe size, Caribbean fishes, Colombia.

Quantitative knowledge of diet and hence trophic level of species is a key piece of information in our understanding of functioning of marine ecosystems. If fisheries are to be managed in the context of the ecosystem, a paramount input is the trophic level of the species involved, e. g., Stergiou et al. (2007); see Cury et al. (2005) and references there in and Vivas-Muñoz et al. (2008) for an application. In neotropical waters knowledge of fish diets is not scarce but the determination of trophic levels has been rarely intended.

The present work provides a list of trophic levels on the basis of diets determined locally in the Colombian Caribbean that may be useful as input for diagnostic tools, e.g. the fish in balance index of Pauly et al. (2000a), and ecotrophic and fishery models, e.g. ECOPATH type models (Christensen \& Pauly 1992, Gascuel 2005). A regression model is proposed that relates size and trophic level as a last resort approach in case of absence of quantitative data of fish diets.

\section{MATERIALS AND METHODS}

A bibliographic search was conducted including journals and thesis works developed for the Colombian Caribbean fish species of economic importance (Fig. 1). Those works with quantitative data (percentage weight) were preferred, although a recent approach may allow converting frequency data into percentage weight (Stobberup et al. 2009). Unidentifiable stomach material was excluded in percentage weight calculations. When a dietary study reported diets by size ranges, a trophic level was calculated for each size range of fish.

Trophic levels, defined as the sum of the trophic levels of prey weighted by their fraction in the predator's diet plus one, were estimated using the application TrophLab (Pauly et al. 2000b). TrophLab allows for three levels of taxonomic resolution of diets and postulates trophic levels for preys in the diets. Thus for each species a list of the typical 


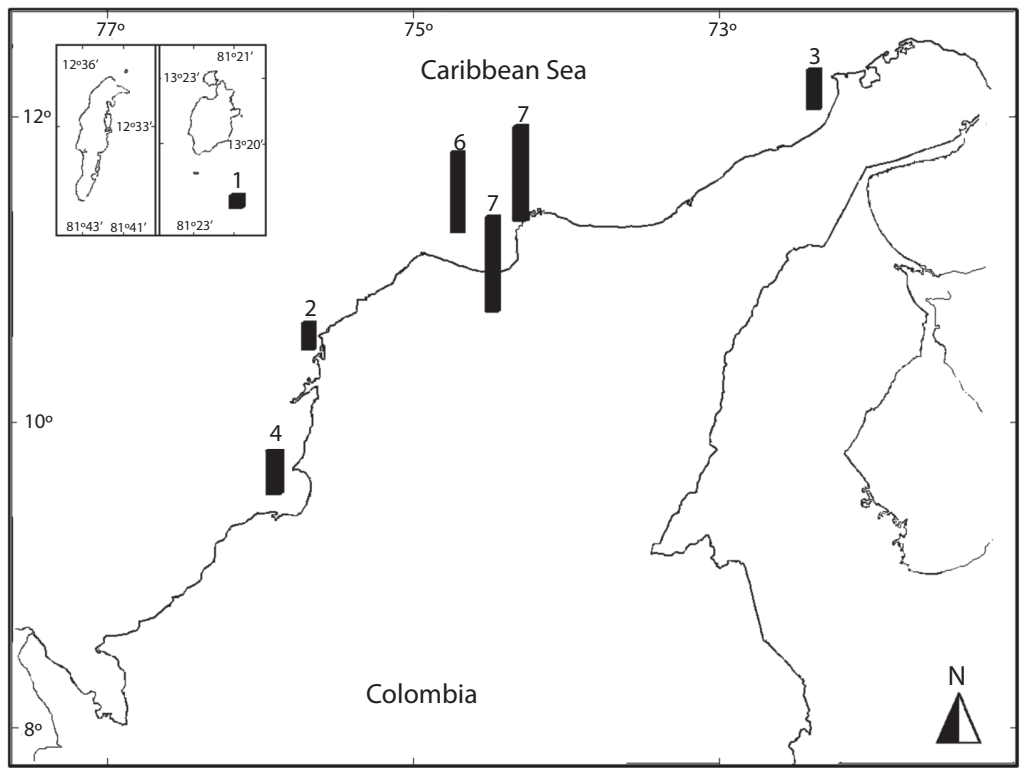

Fig. 1. Spatial distribution of documents on fish diets considered. Numbers on the bars are numbers of documents.

diet was constructed and percentage weight was assigned to items according to constrains in TrophLab.

Ordinary last squares regression was used to relate trophic level with fish size (fish size transformed to natural logarithms as to linearize the relationship). Both maximum length reported and the mean between minimum and maximum length were tested. The last is a crude attempt to give representation of size spectra in the determination of trophic level of a given species. Total length and fork length were transformed to standard length by means of factors obtained from local photographs or from images located in FishBase (Froese \& Pauly 2010) with the exception of elasmobranchs and some bony fishes that lack a caudal fin. Only bony fishes were used in the regression analysis.

\section{RESULTS}

A total of 164 trophic levels for 119 fish species could be estimated (Table 1). These fishes represent about $20 \%$ of marine fishes known to occur in Colombian Caribbean waters (INVEMAR 2010). All fishes of fishery interest are included suggesting a bias towards those fishes. Most fishes have received only one dietary study but some had several (e.g. Caranx crysus, C. hippos, Lutjanus synagris, Megalops atlanticus, among others, Table 1). For those fishes with more than one dietary study estimation of trophic level was found to vary, probably due to a combination of different size ranges, with bigger fish tending to have higher trophic levels (see regression analysis below), and varying sample sizes used (Table 1). One additional source of variance is the taxonomic resolution of the original work.

Figure 2 shows the plot of trophic level (TL) vs. mean standard length (SL, plot of trophic level vs. maximum standard length not shown). The slope of both regressions (after transforming size to natural logarithms) is significantly different from zero $(\mathrm{p}<0.05)$ but the regression trophic level vs. In (mean standard length) explains more variability in the data: $30 \%$ vs. $25 \%$ for the other approach. See Figure 2 for the equation. 
TABLE 1

Trophic levels of 119 Colombian Caribbean fishes

\begin{tabular}{|c|c|c|c|c|}
\hline Species & Trophic Level & Size $(\mathrm{mm})$ & Stomachs & Source \\
\hline Acanthostracion polygonius & 2.33 & $135-200$ & 10 & Navajas (1998) \\
\hline Acanthostracion quadricornis & 2.15 & $130-180$ & 3 & Navajas (1998) \\
\hline Acanthurus chirurgus & 2.36 & $119-240$ & 16 & Navajas (1998) \\
\hline Albula nemoptera & 3.87 & $200-345$ & 34 & Melo (1998) \\
\hline Albula vulpes & 3.71 & $235-360$ & 11 & Melo (1998) \\
\hline Alectis ciliaris & 3.98 & $255-270$ & 2 & Melo (1998) \\
\hline Aluterus monoceros & 2.83 & $224-355$ & 25 & Navajas (1998) \\
\hline Anisotremus surinamensis & 3.68 & NR & 1 & Melo (1998) \\
\hline Anisotremus virginicus & 3.78 & 208 & 1 & Melo (1998) \\
\hline Auxis thazard & 4.50 & $229-320$ & 7 & Moreno (1986) \\
\hline Bagre marinus & 3.59 & $225-285$ & 4 & Melo (1998) \\
\hline \multirow[t]{2}{*}{ Bairdiella ronchus } & 3.78 & $55-191$ & 623 & Torres (1994) \\
\hline & 4.50 & NR & 3 & Jiménez (2008) \\
\hline \multirow[t]{2}{*}{ Balistes vetula } & 3.46 & $168-366$ & 11 & Schiller \& García (2000) \\
\hline & 3.05 & $67-370$ & 76 & Martínez(1990) \\
\hline Balistes capriscus & 3.39 & $125-300$ & 179 & Duarte \& Schiller (1997) \\
\hline Calamus penna & 3.43 & $205-323$ & 68 & Melo (1998) \\
\hline Calamus pennatula & 3.41 & $235-275$ & 6 & Melo (1998) \\
\hline \multirow[t]{5}{*}{ Caranx crysos } & 4.30 & $264-313$ & 3 & Melo (1998) \\
\hline & 4.43 & $179-298$ & 14 & Pinilla (1986) \\
\hline & 4.48 & $306-340$ & 20 & Pinilla (1986) \\
\hline & 4.46 & $349-459$ & 16 & Pinilla (1986) \\
\hline & 4.49 & $157-472$ & 380 & Reyes (1999) \\
\hline \multirow[t]{5}{*}{ Caranx hippos } & 4.50 & $280-290$ & 2 & Melo (1998) \\
\hline & 4.37 & $167-250$ & 50 & Pinilla (1986) \\
\hline & 4.44 & $258-500$ & 11 & Pinilla (1986) \\
\hline & 4.50 & $509-792$ & 14 & Pinilla (1986) \\
\hline & 4.23 & NR & 3 & Jiménez (2008) \\
\hline Caranx latus & 4.41 & $194-235$ & 5 & Melo (1998) \\
\hline Carcharhinus signatus & 4.44 & NR & 3 & Melo (1998) \\
\hline Cathorops mapale & 3.77 & NR & 6 & Jiménez (2008) \\
\hline \multirow[t]{2}{*}{ Cathorops spixii } & 3.67 & $86-343$ & 108 & Santacruz (1989) \\
\hline & 3.28 & $116-207$ & 41 & Melo (1998) \\
\hline \multirow[t]{2}{*}{ Centropomus ensiferus } & 4.28 & $93-160$ & 4 & Bustos (2003) \\
\hline & 4.24 & NR & 8 & Jiménez (2008) \\
\hline \multirow[t]{2}{*}{ Centropomus undecimalis } & 4.07 & $75-162$ & 10 & Bustos (2003) \\
\hline & 4.40 & $114-852$ & 223 & Sierra (1996) \\
\hline Chaetodipterus faber & 3.12 & $130-255$ & 60 & Melo (1998) \\
\hline Chaetodon ocellatus & 3.51 & $70-125$ & 8 & Navajas (1998) \\
\hline Chaetodon sedentarius & 3.41 & $110-130$ & 3 & Navajas (1998) \\
\hline Chilomycterus antillarum & 3.50 & 140 & 1 & Navajas (1998) \\
\hline Chloroscombrus chrysurus & 3.59 & $119-270$ & 38 & Melo (1998) \\
\hline Conodon nobilis & 3.88 & $132-290$ & 103 & Melo (1998) \\
\hline Ctenosciaena gracilicirrhus & 3.92 & $87-190$ & 37 & Navajas (1998) \\
\hline \multirow{2}{*}{ Cynoscion jamaicensis } & 4.40 & NR & 8 & Jiménez (2008) \\
\hline & 4.49 & 205-224 & 3 & Navajas (1998) \\
\hline Dasyatis americana & 3.74 & $670-162 \mathrm{TL}$ & 6 & Navajas (1998) \\
\hline \multirow[t]{2}{*}{ Dasyatis guttata } & 4.02 & 154-710 DW & 19 & Mojica (2007) \\
\hline & 3.43 & 440-1550 TL & 16 & Navajas (1998) \\
\hline \multirow[t]{2}{*}{ Diapterus auratus } & 3.43 & 200 & 1 & Navajas (1998) \\
\hline & 2.97 & $61-182$ & 27 & Arenas \& Acero (1992) \\
\hline Diapterus rhombeus & 2.60 & $56-110$ & 79 & Arenas \& Acero (1992) \\
\hline Diodon holocanthus & 3.36 & $110-210$ & 25 & Navajas (1998) \\
\hline
\end{tabular}


TABLE 1 (Continued)

Trophic levels of 119 Colombian Caribbean fishes

\begin{tabular}{|c|c|c|c|c|}
\hline Species & Trophic Level & Size $(\mathrm{mm})$ & Stomachs & Source \\
\hline Elegatis bippinulata & 4.24 & $260-530$ & 42 & Posada (2005) \\
\hline \multirow[t]{3}{*}{ Elops saurus } & 3.60 & $305-440$ & 2 & Melo (1998) \\
\hline & 4.32 & $192-715$ & 449 & Santos \& Arboleda (1993) \\
\hline & 4.35 & NR & 9 & Jiménez (2008) \\
\hline \multirow[t]{2}{*}{ Eucinostomus argenteus } & 3.75 & $70-154$ & 76 & Navajas (1998) \\
\hline & 3.29 & $97-123$ & 11 & Arenas \&Acero (1992) \\
\hline \multirow[t]{3}{*}{ Eucinostomus gula } & 3.58 & $80-160$ & 63 & Navajas (1998) \\
\hline & 3.15 & $61-101$ & 7 & Arenas \& Acero (1992) \\
\hline & 3.37 & $78-101$ & 68 & Santacruz (1989) \\
\hline Eucinostomus harengulus & 3.22 & $94-110$ & 9 & Arenas \& Acero (1992) \\
\hline Eucinostomus melanopterus & 3.14 & $80-114$ & 23 & Arenas \& Acero (1992) \\
\hline \multirow{2}{*}{ Eugerres plumierii } & 3.08 & $94-266$ & 38 & Navajas (1998) \\
\hline & 3.09 & $78-216$ & 155 & Arenas \& Acero (1992) \\
\hline \multirow[t]{2}{*}{ Euthynnus alletteratus } & 4.47 & $184-643$ & 65 & Moreno (1986) \\
\hline & 4.49 & $240-530$ & 46 & Posada (2005) \\
\hline Fistularia petimba & 4.50 & $105-680$ & 4 & Navajas (1998) \\
\hline Fistularia tabacaria & 4.50 & $636-713$ & 3 & Navajas (1998) \\
\hline \multirow[t]{3}{*}{ Gerres cinereus } & 3.23 & $155-255$ & 2 & Navajas (1998) \\
\hline & 3.04 & $62-139$ & 54 & Arenas \& Acero (1992) \\
\hline & 3.46 & $65-139$ & 23 & Santacruz (1989) \\
\hline Haemulon album & 3.71 & 320 & 1 & Melo (1998) \\
\hline Haemulon aurolineatum & 3.58 & $86-235$ & 24 & Melo (1998) \\
\hline Haemulon bonariense & 4.26 & NR & 6 & Jiménez (2008) \\
\hline Haemulon melanurum & 3.42 & $60-235$ & 3 & Melo (1998) \\
\hline \multirow[t]{2}{*}{ Haemulon plumierii } & 3.82 & $104-285$ & 155 & Rojas \& Botero (1987) \\
\hline & 3.27 & $10-245$ & 5 & Melo (1998) \\
\hline Haemulon steindachneri & 3.57 & $80-285$ & 90 & Melo (1998) \\
\hline Hemicaranx amblyrhynchus & 3.80 & $135-299$ & 2 & Melo (1998) \\
\hline Holocentrus ascensionis & 3.47 & $180-220$ & 7 & Navajas (1998) \\
\hline Isopisthus parvipinnis & 4.50 & NR & 6 & Jiménez (2008) \\
\hline Larimus breviceps & 4.38 & 110-195 & 14 & Navajas (1998) \\
\hline Lepophidium profundorum & 3.92 & $100-280 \mathrm{TL}$ & 72 & Santacruz (1989) \\
\hline \multirow[t]{2}{*}{ Lutjanus analis } & 4.10 & 210 a 460 & 110 & Duarte \& García (1999a) \\
\hline & 4.04 & NR & 126 & Arévalo (1996) \\
\hline Lutjanus apodus & 4.11 & $184-334$ & 3 & Navajas (1998) \\
\hline Lutjanus griseus & 4.50 & 426 & 1 & Navajas (1989) \\
\hline Lutjanus jocu & 4.42 & $260-525$ & 10 & Navajas (1989) \\
\hline Lutjanus mahogoni & 4.47 & $200-225$ & 4 & Navajas (1989) \\
\hline Lutjanus purpureus & 3.59 & 150 & 1 & Navajas (1989) \\
\hline \multirow[t]{5}{*}{ Lutjanus synagris } & 3.83 & $110-320$ & 94 & Duarte \& García 1999b) \\
\hline & 3.92 & $87-301$ & 106 & Martínez (1990) \\
\hline & 4.00 & $86-249$ & 91 & Santacruz (1989) \\
\hline & 4.10 & NR & 116 & Arévalo (1996) \\
\hline & 4.06 & NR & 4 & Jiménez (2008) \\
\hline Lutjanus vivanus & 3.93 & $102-290$ & 7 & Navajas (1998) \\
\hline \multirow{4}{*}{ Megalops atlanticus } & 4.16 & $305-470$ & 2 & Navajas (1989) \\
\hline & 4.13 & $93-205$ & 101 & Cataño \& Garzón (1994) \\
\hline & 4.29 & $206-317$ & 93 & Cataño \& Garzón (1994) \\
\hline & 4.43 & $318-495$ & 108 & Cataño \& Garzón (1994) \\
\hline Menticirrhus littoralis & 4.09 & $227-244$ & 4 & Navajas (1998) \\
\hline \multirow{2}{*}{ Micropogonias furnieri } & 3.23 & $63-325$ & 246 & Escobar (1994) \\
\hline & 3.98 & $167-353$ & 29 & Navajas (1998) \\
\hline Mugil curema & 2.00 & $67-194$ & 276 & Bustos et al. (2004) \\
\hline Mugil incilis & 2.23 & $60-293$ & 15 & Velasco (1985) \\
\hline
\end{tabular}


TABLE 1 (Continued)

Trophic levels of 119 Colombian Caribbean fishes

\begin{tabular}{|c|c|c|c|c|}
\hline Species & Trophic Level & Size (mm) & Stomachs & Source \\
\hline Mustelus canis & 3.87 & $265-487$ & 8 & Melo (1998) \\
\hline Myripristis jacobus & 3.51 & $125-155$ & 9 & Navajas (1998) \\
\hline Narcine bancroftii & $3.60^{*}$ & 109-594 TL & 79 & Moreno et al. (2009) \\
\hline Narcine brasiliensis & 3.55 & $240-470 \mathrm{TL}$ & 8 & Melo (1998) \\
\hline Ocyurus chrysurus & 4.31 & $75-376$ & 123 & Rojas \& Botero (1987) \\
\hline Oligoplites palometa & 4.45 & $56-280$ & 86 & Duque et al. (1996) \\
\hline Oligoplites saurus & 4.48 & $60-249$ & 282 & Duque et al. 1996) \\
\hline \multirow[t]{2}{*}{ Opisthonema oglinum } & 2.36 & NR & 12 & Jiménez (2008) \\
\hline & 3.30 & $34-288$ & 64 & Navajas (1998) \\
\hline \multirow[t]{2}{*}{ Pellona harroweri } & 3.37 & $34-91$ & 21 & Críales (2003) \\
\hline & 3.67 & $67-221$ & 15 & Navajas (1998) \\
\hline Polydactylus virginicus & 3.90 & $140-222$ & 19 & Navajas (1998) \\
\hline Pomacanthus paru & 2.03 & 114 & 1 & Navajas (1998) \\
\hline Pomadasys corvinaeformis & 3.35 & $96-400$ & 44 & Melo (1998) \\
\hline Pomadasys crocro & 3.50 & NR & 1 & Melo (1998) \\
\hline Priacanthus arenatus & 3.89 & $130-290$ & 16 & Melo (1998) \\
\hline Prionotus punctatus & 3.74 & $145-240$ & 2 & Melo (1998) \\
\hline Pristipomoides aquilonaris & 4.14 & $150-200$ & 6 & Navajas (1998) \\
\hline \multirow{2}{*}{ Pseudopeneus maculatus } & 3.76 & $69-147$ & 74 & Santacruz (1989) \\
\hline & 4.05 & $104-190$ & 14 & Melo (1998) \\
\hline Rhinesomus bicaudalis & 3.14 & $180-280$ & 2 & Navajas (1998) \\
\hline Rhinobatos percellens & 3.70 & $319-575$ & 24 & Melo (1998) \\
\hline Rhinoptera bonasus & 3.59 & 554 & 1 & Melo (1998 \\
\hline Rhomboplites aurorubens & 4.20 & $150-205$ & 7 & Navajas (1998) \\
\hline Saurida normani & 4.46 & $155-237$ & 3 & Navajas (1998) \\
\hline \multirow{3}{*}{ Scomberomorus brasiliensis } & 4.45 & $295-605$ & 11 & Navajas (1998) \\
\hline & 4.50 & $167-583$ & 56 & Moreno (1986) \\
\hline & 4.50 & NR & 5 & Jiménez (2008) \\
\hline Scomberomorus cavalla & 4.50 & $270-946$ & 11 & Moreno (1986) \\
\hline Scorpaena plumieri & 3.30 & $165-224$ & 9 & Melo (1998) \\
\hline \multirow[t]{4}{*}{ Selar crumenophthalmus } & 3.97 & $150-257$ & 37 & Melo (1998) \\
\hline & 4.13 & $182-191$ & 23 & Pinilla (1986) \\
\hline & 4.09 & $201-218$ & 57 & Pinilla (1986) \\
\hline & 4.30 & $226-261$ & 13 & Pinilla (1986) \\
\hline Selene brownii & 4.38 & $95-280$ & 39 & Melo (1998) \\
\hline Selene setapinnis & 3.60 & $120-210$ & 8 & Melo (1998) \\
\hline Selene vomer & 3.97 & $145-304$ & 39 & Melo (1998) \\
\hline Seriola rivoliana & 4.34 & $220-260$ & 3 & Melo (1998) \\
\hline Seriola zonata & 3.54 & 255 & 1 & Melo (1998) \\
\hline Serranus atrabranchus & 4.01 & 70 & 1 & Melo (1998) \\
\hline Sphoeroides greeleyi & 3.09 & $35-102$ & 337 & Londoño (1994) \\
\hline Sphoeroides testudineus & 3.35 & $52-210$ & 329 & Londoño (1994) \\
\hline Sphyraena barracuda & 4.50 & $>427$ & 51 & Bent (2006) \\
\hline Sphyraena guachancho & 4.40 & 285.443 & 5 & Navajas (1998) \\
\hline Sphyraena picudilla & 4.48 & $215-362$ & 6 & Navajas (1998) \\
\hline Stellifer griseus & 4.50 & NR & 6 & Jiménez (2008) \\
\hline Stephanolepis setifer & 2.62 & 137 & 1 & Navajas (1998) \\
\hline Syacium micrurum & 3.50 & $105-130$ & 3 & Navajas (1998) \\
\hline Thunnus atlanticus & 4.48 & $176-748$ & 26 & Moreno (1986) \\
\hline Trachinotus carolinus & 3.50 & 340 & 1 & Melo (1998) \\
\hline Trachinotus falcatus & 3.23 & $370-393$ & 2 & Melo (1998) \\
\hline \multirow[t]{2}{*}{ Trichurus lepturus } & 4.47 & $490-1120 \mathrm{TL}$ & 12 & Melo (1998) \\
\hline & 4.50 & NR & 5 & Jiménez (2008) \\
\hline Umbrina broussonetti & 4.07 & $145-210$ & 15 & Navajas (1998) \\
\hline
\end{tabular}


TABLE 1 (Continued)

Trophic levels of 119 Colombian Caribbean fishes

$\begin{array}{rcccc}\text { Species } & \text { Trophic Level } & \text { Size }(\mathrm{mm}) & \text { Stomachs } & \text { Source } \\ \text { Umbrina coroides } & 3.92 & \text { NR } & 4 & \text { Jiménez (2008) } \\ & 3.44 & 131-205 & 84 & \text { Navajas (1998) } \\ \text { Upeneus parvus } & 4.28 & 160-175 & 3 & \text { Melo (1998) }\end{array}$

Unless otherwise stated size measure is standard length. NR=Not reported, TL=Total length, DW=Disk width. * As reported in Moreno et al. (2009).

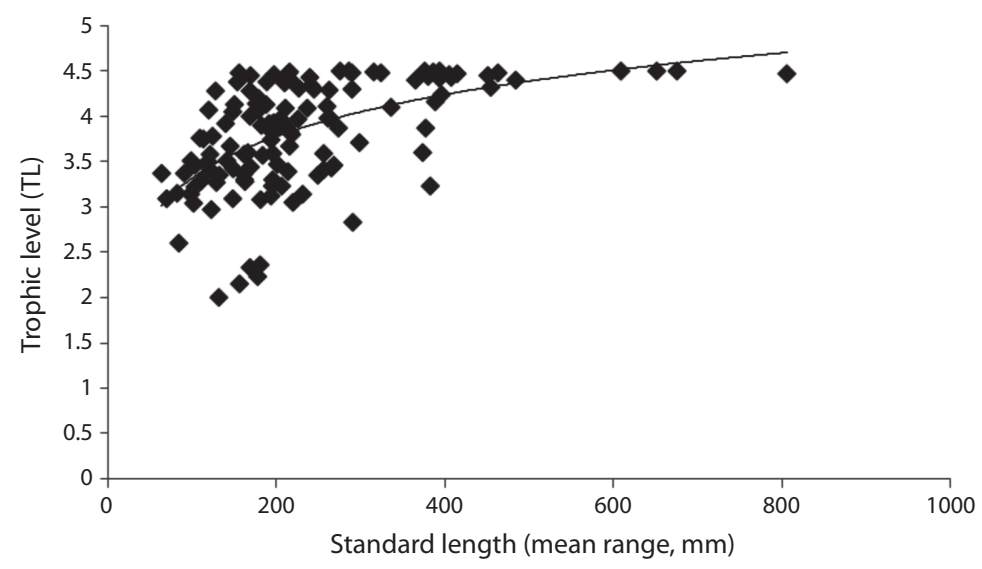

Fig. 2. Relationship between mean range standard length (SL) and trophic level (TL) in some Colombian Caribbean bony fishes. Regression equation was: $\mathrm{TL}=0.2678+0.6622 * \ln ($ mean $\mathrm{SL}), \mathrm{r}^{2}=0.30, \mathrm{p}<0.05$.

\section{DISCUSSION}

Much work remains to be done. Spatial coverage of studies is quite uneven with the bulk of the work done in the central Colombian Caribbean thus spatial cover must be improved as spatial comparisons would be informative. It is unfortunate that a substantial amount of work has not been published as it is contained in gray literature (thesis works). No work has attempted to follow temporal changes in diets and only few have come close to size-based changes in diets. However, our results suggest that trophic level increases with size for a given species (Table 1).

The trophic levels reported here should be viewed with care. Size structure should be taken into account and be given adequate weight in calculations. A weakness in the regression model presented here as a predictive tool is the uneven number of stomachs of fishes size range. Clearly, size is not everything as it explains a relative low percentage in trophic level variation (via a logarithmic relationship), although it may be the single most important factor. For instance, Romanuk et al. (2010), using a much bigger data set, found that fish size explained about $20 \%$ of fish trophic level variation, while phylogeny explained an additional $17 \%$. Nevertheless, it is hoped that both the trophic level list and the regression presented here turns out to be useful to researchers in the neotropics and elsewhere. 


\section{ACKNOWLEDGMETS}

Comments by two anonymous referees helped to improve de paper.

\section{RESUMEN}

Estudios ecológicos de especies de peces importantes comercialmente son de gran valor como insumos en temas de manejo. Aquí se presenta estimaciones del nivel trófico de aquellas especies de peces del Caribe colombiano cuya dieta ha sido descrita localmente. Información utilizable sobre 119 especies resulto en 164 estimaciones de nivel trófico. Se propone un modelo de regresión ordinaria entre el nivel trófico y el tamaño de los peces. La pendiente resultó positiva y significativamente diferente de cero $(\mathrm{p}<0.05)$ lo cual sugiere una relación moduladora entre el nivel trófico y el tamaño de los peces. Tanto la lista de niveles tróficos como el modelo de regresión, deben ser de ayuda en la formulación de indicadores tróficos y modelos de los ecosistemas neotropicales.

Palabras clave: nivel trófico, talla de peces, peces del Caribe, Colombia.

\section{REFERENCES}

Arenas, P. \& A. Acero. 1992. Organización trófica de las mojarras (Pisces:Gerreidae) de la Ciénaga Grande de Santa Marta (Caribe Colombiano). Rev. Biol. Trop. 40: 28-302.

Arévalo, J.C. 1996. Caracterización trófica y reproductiva de las poblaciones de Lutjanus analis (Cuvier 1828) y Lutjanus synagris (Linnaeus 1758) en el Caribe Colombiano. Tesis de grado, Universidad Jorge Tadeo Lozano, Bogotá, Colombia.

Bent, H.C. 2006. Biología, ecología y pesquería de la Barracuda Sphyraena Barracuda (Walbaum 1972) (Pisces: Perciformes:Sphyraenidae) en la Isla de San Andrés y los Cayos Bolívar y Albuquerque, Caribe Colombiano. Tesis de grado, Universidad Jorge Tadeo Lozano, Bogotá, Colombia.

Bustos, D., D. Pérez, C.B. García \& A. Sanjuán. 2004. Efecto potencial de procesos hidrológicos en la dieta y la condición de Mugil curema (Mugilidae), en la laguna de Navío Quebrado, La Guajira, Caribe colombiano, p. 235-254. In N. Campo \& A. Acero (eds). Contribuciones en ciencias del mar en Colombia. Investigación y desarrollo de territorios promisorios. REMAR, Unibiblos, Bogotá, Colombia.

Bustos, D.M. 2003. Ecología trófica y algunos aspectos biológicos de las especies pertenecientes a las familias Mugilidae y Centropomidae en la laguna de Navío Quebrado, Guajira, Caribe Colombiano. Tesis de grad, Universidad Jorge Tadeo Lozano, Bogotá, Colombia.

Cataño, S. \& J. Garzón. 1994. Ecología trófica del sábalo Megalops atlanticus (Pisces: Megalopidae) en el área de la Ciénaga Grande de Santa Marta, Caribe colombiano. Rev. Biol. Trop. 42: 673-684.

Christenesen, V. \& D. Pauly. 1992. ECOPATH II - a software for balancing steady-state ecosystem models and calculating network characteristics. Ecol. Mod. 61: 169-185.

Críales, M.I. 2003. Composición de la dieta de Pellona harroweri (Pisces: Pristigasteridae) en la Guajira Caribe colombiano. Bol. Invest. Mar. Cost. 32: 279-282.

Cury, P.M., L.J. Shannon, J.P. Roux, G.M. Daskalov, A. Jarre, C.L. Moloney \& D. Pauly. 2005. Trophodynamic indicators for an ecosystem approach to fisheries. ICES J. Mar. Sci. 62: 430-442.

Duarte L.O. \& C.B. García. 1999b. Diet of the Lane Snapper, Lutjanus synagris (Lutjanidae), in the Gulf of Salamanca, Colombia. Caribbean J. Sci. 35: 54-63.

Duarte, L.O. \& C.B. García. 1999a. Diet of the Mutton Snapper Lutjanus analis (Cuvier) from the Gulf of Salamanca, Colombia Caribbean. Bull. Mar. Sci. 65: 453-465.

Duarte, L.O. \& D. Von Schiller. 1997. Comunidad de peces demersales del Golfo de Salamanca (Caribe Colombiano), estructura espacio-temporal y caracterización trofica con énfasis en los hábitos alimenticios de Lutjanus analis (Cuvier 1828), Lutjanus synagris (Linnaeus 1758), Balistes capriscus (Gmelin 1788) y Balistes vetula (Linnaeus 1758). Tesis de grado, Universidad Jorge Tadeo Lozano, Bogotá, Colombia.

Duque, G., A. Acero, A. Santos \& E. Rubio. 1996. Food habits of the species of the genus Oligoplites (Carangidae) from the Ciénaga Grande de Santa Marta, Colombian Caribbean. Cybium 20: 251-260.

Escobar, M.M. 1994. Aspectos biológicos y ecológicos, con énfasis en reproducción y alimentación, de Micropogonias furnieri (Desmarest) y Stellifer venezuelae (Schultz) (Pisces: Scianidae), Ciénaga Grande de Santa Marta, Caribe Colombiano. Tesis de grado, Pontificia Universidad Javeriana, Bogotá, Colombia.

Froese, R. \& D. Pauly. Editors. 2010. FishBase World Wide Web electronic publication. (Downloaded: May 30, 2010, www.fishbase.org, version 11/2010). 
Gascuel, D. 2005. The trophic-level based model: A theoretical approach of fishing effects on marine ecosystems. Ecol. Mod. 189: 315-332.

INVEMAR (2010). Sistema de información sobre biodiversidad marina de Colombia (Downloaded: May 30, 2010, http://siam.invemar.org.co/siam/sibm/index.htm).

Jiménez, M.F. 2008. Caracterización trófica de algunos peces de interés comercial en la bahía de Cartagena, Caribe Colombiano. Tesis de grado, Universidad Jorge Tadeo Lozano, Bogotá, Colombia.

Londoño, S. 1994. Ecología trófica y aspectos reproductivos de Sphoeroides greeleyi Gilbert 1900 y Sphoeroides testudineus (Linnaeus 1758) en la Ciénaga Grande de Santa Marta, Caribe Colombiano. Tesis de grado, Pontificia Universidad Javeriana, Bogotá, Colombia.

Martínez, J. 1990. Aspectos bioecológicos del pargo chino Lutjanus synagris Linnaeus, 1758, y el pejepuerco Balistes vetula (Linnaeus 1758), en arrecifes del archipiélago de San Bernardo, Mar Caribe colombiano. Tesis de grado, Universidad Nacional de Colombia, Bogotá, Colombia.

Melo, G. 1998. Caracterización trófica de los peces capturados con red de arrastre demersal en el Golfo de Salamanca, Caribe Colombiano parte 1. Tesis de grado, Pontificia Universidad Javeriana, Bogotá, Colombia.

Mojica, D.F. 2007. Bioecología de la raya látigo Dasyatis guttata (Bloch \& Schneider 1801) capturada con artes de pesca artesanal en Don Jaca, Santa Marta Caribe Colombiano. Tesis de grado, Universidad Jorge Tadeo Lozano, Bogotá, Colombia.

Moreno, F., K. Acevedo, M. Grijalba-Bendek, C. PoloSilva \& A. Acero. 2009. Espectro trófico de la raya eléctrica Narcine bancroftii (Griffith \& Smith 1834) en playa Salguero, Santa Marta, Caribe Colombiano. Pan-American J. Aquatic Sci. 4: 413-422.

Moreno, R. 1986. Ecología trófica de algunas especies de la familia Scombridae capturados en aguas costeras del departamento del Magdalena, Caribe Colombiano. Tesis de grado, Universidad Nacional de Colombia, Bogotá, Colombia.

Navajas, P. 1998. Caracterización trófica de los peces capturados con red de arrastre demersal en el Golfo de Salamanca, Caribe Colombiano parte II. Tesis de grado, Pontificia Universidad Javeriana, Bogotá, Colombia.

Pauly, D., R. Froese, P. Sa-a, M.L. Palomares, V. Christensen \& J. Rius. 2000b. TrophLab in MS Access. (Downloaded: May 30, 2010, www.fishbase.org/ download/TrophLab2K.zip).
Pauly, D., V. Christensen \& C. Walters. 2000a. Ecopath, Ecosim, and Ecospace as tools for evaluating ecosystem impact of fisheries. ICES J. Mar. Sci. 57: 697-706.

Pinilla, G. 1986. Ecología trófica del jurel, Caranx hippos (Linnaeus 1766), La cojinoa, Caranx crysos Mitchil, 1815, y el ojo gordo, Selar crumenophthalmus, (Bloch 1793), Pisces Carangidae, en aguas costeras del departamento del Magdalena, Caribe Colombiano. Tesis de grado, Universidad Nacional de Colombia, Bogotá, Colombia.

Posada, M.C. 2005. Ecología trófica de Elagatis bipinnulata (Quoy \& Gaimard 1824) (Pisces: Carangidae) y Euthynnus alletteratus (Rafinesque 1810) (Pisces: Scombridae), en la región de Taganga y Parque Nacional Natural Tayrona, Caribe colombiano. Tesis de grado, Universidad Jorge Tadeo Lozano, Bogotá, Colombia.

Reyes, A.A. 1999. Hábitos alimentarios y algunos aspectos reproductivos de la Cojinoa Negra Caranx crysos (Mitchill 1815). Tesis de grado, Universidad Jorge Tadeo Lozano, Bogotá, Colombia.

Rojas, G.C. \& I. Botero. 1987. Aspectos bioecológicos de la saltona, Ocyurus chrysurus (Bloch 1791) y del ronco blanco Haemulon plumieri (Lacepede 1802) en arrecifes del archipiélago de San Bernardo, mar Caribe Colombiano. Tesis de grado, Universidad Nacional de Colombia, Bogotá, Colombia.

Romanuk, T.N., A. Hayward \& J.A. Hutchings. 2011. RESEARCH PAPER: Trophic level scales positively with body sizes in fishes. Global. Ecol. Biogeogr. no. doi. (Consultado 11 de febrero 2011, http://onlinelibrary.wiley.com/ doi/10.1111/j.1466-8238.2010.00579.x/full)

Santacruz, A.R. 1989. Contribución al estudio ecológicopesquero de la fauna ictica acompañante en la pesca de arrastre de camarón por barcos arrastreros en el Golfo de Morrosquillo, Mar Caribe Colombiano. Tesis de grado, Universidad Nacional de Colombia, Bogotá, Colombia

Santos, A. \& S. Arboleda. 1993. Aspectos biológicos y ecológicos del macabí Elops saurus Linnaeus (Pisces: Elopidae) en la Ciénaga Grande de Santa Marta y costa adyacente, Caribe colombiano. Bol. Invest. Mar. Cost. 22: 77-96.

Shiller, D. \& C.B. García. 2000. Observations on the diet of Balistes vetula (Pisces: Balistidae) in the Gulf of Salamanca Colombian Caribbean. Bol. Invest. Mar. Cost. 29: 35-40. 
Sierra, P.C. 1996. Biología, ecología y algunos aspectos pesqueros del róbalo Centropomus undecimales. Tesis de grado, Universidad Jorge Tadeo Lozano, Bogotá, Colombia.

Stergiou, K.I., D.K. Moutopoulos, J.A. Hernando-Casal \& K. Erzini. 2007. Trophic signatures of small-scale fishing gears and their implications for conservation and management. Mar. Ecol. Prog. Ser. 333: 117-28.

Stobberup, K.A., T. Morato, P. Amorim \& K. Erzini. 2009. Predicting weight composition of fish diets: Converting frequency of occurrence of prey to relative weight composition. The Open Fish Sci. J. 2: 42-49.
Torres, L. 1994. Aspectos biológicos y ecológicos de la carrura Bairdiella ronchus (Cuvier 1830) (Pisces Sciaenidae) en la Ciénaga Grande de Santa Marta, Caribe colombiano. Tesis de grado, Pontificia Universidad Javeriana, Bogotá, Colombia.

Velasco, A. 1985. Aportes al conocimiento de la historia de vida de Mugil incilis Hancock 1830. Tesis de grado, Universidad Jorge Tadeo Lozano, Bogotá, Colombia.

Vivas-Muñoz, J.C., L.O. Duarte \& C.B. García. 2008. Exploración de tendencias históricas de indicadores trofodinámicos en los peces demersales del Mar Caribe de Colombia. Proc. Gulf Caribb. Fish. Institute 60: 338-344. 